\title{
Delayed Feedback Control and Bifurcation Analysis of an Autonomy System
}

\author{
Zhen Wang, ${ }^{1}$ Huitao Zhao, ${ }^{2,3}$ and Xiangyu Kong ${ }^{1}$ \\ ${ }^{1}$ Institute of Information and System Computation Science, Beifang University of Nationalities, Yinchuan, Ningxia 750021, China \\ ${ }^{2}$ Department of Mathematics and Information Science, Zhoukou Normal University, Zhoukou, Henan 466001, China \\ ${ }^{3}$ Department of Applied Mathematics, Kunming University of Science and Technology, Kunming, Yunnan 650093, China
}

Correspondence should be addressed to Zhen Wang; jen715@163.com

Received 28 February 2013; Accepted 26 March 2013

Academic Editor: Yisheng Song

Copyright (C) 2013 Zhen Wang et al. This is an open access article distributed under the Creative Commons Attribution License, which permits unrestricted use, distribution, and reproduction in any medium, provided the original work is properly cited.

\begin{abstract}
An autonomy system with time-delayed feedback is studied by using the theory of functional differential equation and Hassard's method; the conditions on which zero equilibrium exists and Hopf bifurcation occurs are given, the qualities of the Hopf bifurcation are also studied. Finally, several numerical simulations are given; which indicate that when the delay passes through certain critical values, chaotic oscillation is converted into a stable state or a stable periodic orbit.
\end{abstract}

\section{Introduction}

Since the first chaotic attractor was found by Lorsenz in 1963, the theory of chaos has been developing rapidly. The topics of chaos and chaotic control are growing rapidly in many different fields such as biological systems and ecological and chemical systems [1-5]. The desirability of chaos depends on the particular application. Therefore, it is important that the chaotic response of a system can be controlled.

Many researchers have proposed chaos control and synchronization schemes in recent years [6-11]; among which, delayed feedback controller (DFC) is an effective method for chaos control; it has been receiving considerable attention recently [11-14]. The basic idea of DFC is to realize a continuous control for a dynamical system by applying a feedback signal which is proportional to the difference between the dynamical variable $X(t)$ and its delayed value [14]. Choosing the appropriate feedback strength and time delay can make the system stable. The delayed feedback control method does not require any computer analysis and can be simply implemented in various experiments. For example, Song and Wei in [15] investigated the chaos phenomena of Chen's system using the method of delayed feedback control. Their results show that when $K$ taking some value, taking the delay $\tau$ as the bifurcation parameter when $\tau$ passes through a certain critical value, the stability of the equilibrium will be changed from unstable to stable, chaos vanishes and a periodic solution emerges.

Let us consider a system of nonlinear autonomous differential equations as follows:

$$
\begin{gathered}
\dot{x}=a x-y, \\
\dot{y}=x-b y-z, \\
\dot{z}=a x+4 z(y-2) .
\end{gathered}
$$

When $a=0.2, b=0.02$, the zero equilibrium of system (1) is unstable and system (1) is chaotic (see Figure 1). For control of chaos, we add a time-delayed force $K(y(t)-y(t-\tau))$ to the second equation of system (1), that is, the following delayed feedback control system:

$$
\begin{gathered}
\dot{x}=a x-y, \\
\dot{y}=x-b y-z+K(y(t)-y(t-\tau)), \\
\dot{z}=a x+4 z(y-2) .
\end{gathered}
$$

When $K=0$ or $\tau=0$, we can see that system (2) is the same as system (1). In this paper, by taking $\tau$ as bifurcation parameter, we will show that when $K$ takes some value, with 

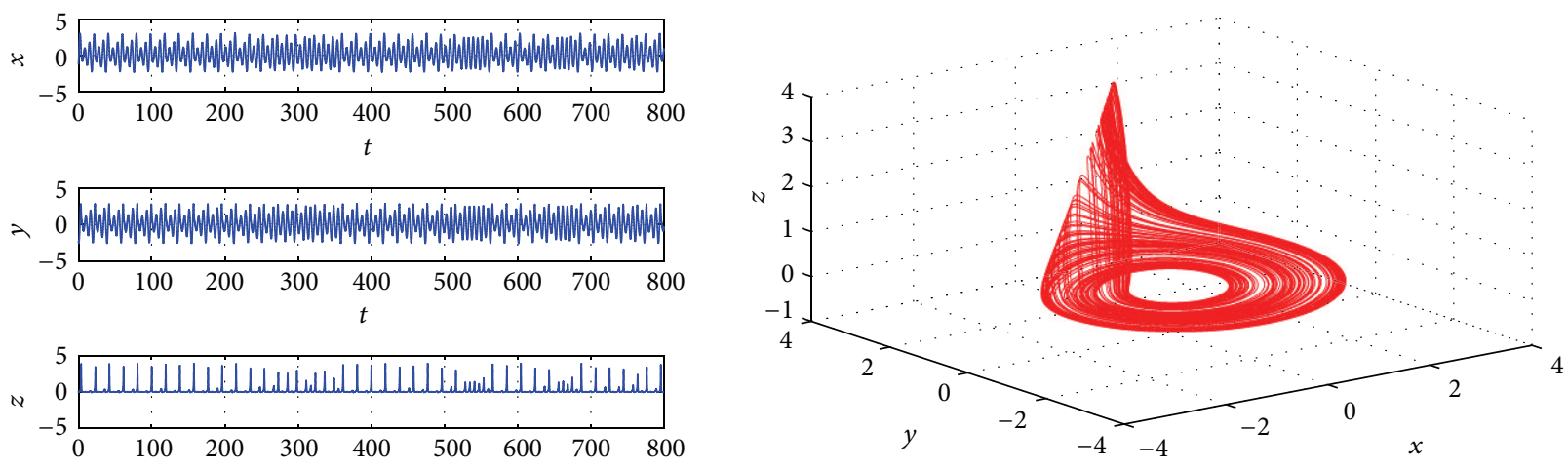

FIGURE 1: The trajectories and phase graphs of system (1) with $a=0.2$ and $b=0.02$.

the increasing of $\tau$, the stability of zero equilibrium of system (2) will change, and a family of periodic orbits bifurcates from zero equilibrium.

This paper is organized as follows. In Section 2, we first focus on the stability and Hopf bifurcation of the zero equilibrium of system (2). In Section 3, we derive the direction and stability of Hopf bifurcation by using normal form and central manifold theory. Finally in Section 4, an example is given for showing the effects of chaotic control.

\section{Local Stability and Delay-Induced Hopf Bifurcations}

In this section, by analyzing the characteristic equation of the linearized system of system (2) at the equilibriums, we investigate the stability of the equilibriums and the existence of local Hopf bifurcations occurring at the equilibriums.

System (2) has two equilibriums, $E_{0}(0,0,0)$ and $E^{*}\left(x^{*}\right.$, $\left.y^{*}, z^{*}\right)$, where

$$
x^{*}=\frac{8(1-a b)-a}{4 a(1-a b)}, \quad y^{*}=a x^{*}, \quad z^{*}=(1-a b) x^{*} .
$$

The linearization of system (2) at $E_{0}$ is

$$
\begin{gathered}
\dot{x}=a x-y, \\
\dot{y}=x-b y-z+K(y(t)-y(t-\tau)), \\
\dot{z}=a x-8 z,
\end{gathered}
$$

whose characteristic equation is

$$
\lambda^{3}+a_{2} \lambda^{2}+a_{1} \lambda+a_{0}+\left(b_{2} \lambda^{2}+b_{1} \lambda+b_{0}\right) e^{-\lambda \tau}=0,
$$

where $a_{2}=8+b-a-K, a_{1}=8 b-8 K-a b+a K-8 a+1, a_{0}=$ $8 a K-8 a b-a+8, b_{2}=K, b_{1}=(8-a) K, b_{0}=-8 a K$, and $\sum_{i=0}^{2} b_{i}^{2} \neq 0$. Thus, we need to study the distribution of the roots of the third-degree exponential polynomial equation. For this end, we first introduce the following simple result which was proved by Ruan and Wei [16] using Rouche's theorem.
Lemma 1. Consider the exponential polynomial

$$
\begin{aligned}
P( & \left.\lambda, e^{-\lambda \tau_{1}}, \ldots, e^{-\lambda \tau_{m}}\right) \\
= & \lambda^{n}+p_{1}^{(0)} \lambda^{n-1}+\cdots+p_{n-1}^{(0)} \lambda+p_{n}^{(0)} \\
& +\left[p_{1}^{(1)} \lambda^{n-1}+\cdots+p_{n-1}^{(1)} \lambda+p_{n}^{(1)}\right] e^{-\lambda \tau_{1}} \\
& +\cdots+\left[p_{1}^{(m)} \lambda^{n-1}+\cdots+p_{n-1}^{(m)} \lambda+p_{n}^{(m)}\right] e^{-\lambda \tau_{m}}
\end{aligned}
$$

where $\tau_{i} \geq 0(i=1,2, \ldots, m)$ and $p_{j}^{(i)}(i=0,1, \ldots, m ; j=$ $1,2, \ldots, n)$ are constants. As $\left(\tau_{1}, \tau_{2}, \ldots, \tau_{m}\right)$ vary, the sum of the order of the zeros of $P\left(\lambda, e^{-\lambda \tau_{1}}, \ldots, e^{-\lambda \tau_{m}}\right)$ on the open right half-plane can change only if a zero appears on or crosses the imaginary axis.

Obviously, $i \omega(\omega>0)$ is a root of (5) if and only if $\omega$ satisfies

$$
\begin{aligned}
-\omega^{3} i & -a_{2} \omega^{2}+a_{1} \omega i+a_{0} \\
& +\left(-b_{2} \omega^{2}+b_{1} \omega i+b_{0}\right)(\cos \omega \tau-\sin \omega \tau)=0 .
\end{aligned}
$$

Separating the real and imaginary parts, we have

$$
\begin{aligned}
& a_{2} \omega^{2}-a_{0}=\left(b_{0}-b_{2} \omega^{2}\right) \cos \omega \tau+b_{1} \omega \sin \omega \tau, \\
& -\omega^{3}+a_{1} \omega=\left(b_{0}-b_{2} \omega^{2}\right) \sin \omega \tau-b_{1} \omega \cos \omega \tau,
\end{aligned}
$$

which is equivalent to

$$
\begin{aligned}
\omega^{6}+ & \left(a_{2}^{2}-b_{2}^{2}-2 a_{1}\right) \omega^{4} \\
& +\left(a_{1}^{2}-2 a_{0} a_{2}-b_{1}^{2}+2 b_{0} b_{2}\right) \omega^{2}+a_{0}^{2}-b_{0}^{2}=0 .
\end{aligned}
$$

Let $z=\omega^{2}$, and then (9) becomes

$$
z^{3}+p z^{2}+q z+r=0
$$

where $p=a_{2}^{2}-b_{2}^{2}-2 a_{1}, q=a_{1}^{2}-2 a_{0} a_{2}-b_{1}^{2}+2 b_{0} b_{2}$, and $r=a_{0}^{2}-b_{0}^{2}$.

Denote

$$
h(z)=z^{3}+p z^{2}+q z+r .
$$

Then, we have the following lemma. 
Lemma 2. For the polynomial equation (10), one has the following results.

(i) If $r<0$, then (10) has at least one positive root.

(ii) If $r \geq 0$ and $\Delta=p^{2}-3 q \leq 0$, then (10) has no positive roots.

(iii) If $r \geq 0$ and $\Delta=p^{2}-3 q>0$, then (10) has positive roots if and only if $z_{1}^{*}=(1 / 3)(-p+\sqrt{\triangle})>0$ and $h\left(z_{1}^{*}\right) \leq 0$.

Suppose that (10) has positive roots, without loss of generality; we assume that it has three positive roots, defined by $z_{1}, z_{2}$, and $z_{3}$, respectively. Then (9) has three positive roots

$$
\omega_{1}=\sqrt{z_{1}}, \quad \omega_{2}=\sqrt{z_{2}}, \quad \omega_{3}=\sqrt{z_{3}} .
$$

Thus, we have

$$
\cos \omega \tau=\frac{b_{1} \omega^{2}\left(\omega^{2}-a_{1}\right)-\left(a_{2} \omega^{2}-a_{0}\right)\left(b_{2} \omega^{2}-b_{0}\right)}{\left(b_{2} \omega^{2}-b_{0}\right)^{2}+b_{1} \omega^{2}} .
$$

If we denote

$$
\begin{gathered}
\tau_{k}^{(j)}=\frac{1}{\omega_{k}}\left\{\arccos \left(\frac{b_{1} \omega^{2}\left(\omega^{2}-a_{1}\right)-\left(a_{2} \omega^{2}-a_{0}\right)\left(b_{2} \omega^{2}-b_{0}\right)}{\left(b_{2} \omega^{2}-b_{0}\right)^{2}+b_{1} \omega^{2}}\right)\right. \\
+2 j \pi, k=1,2,3 ; j=0,1, \ldots\},
\end{gathered}
$$

then $\pm i \omega_{k}$ is a pair of purely imaginary roots of (9) with $\tau_{k}^{(j)}$. Define

$$
\tau_{0}=\tau_{k_{0}}^{(0)}=\min _{k \in\{1,2,3\}}\left\{\tau_{k}^{(0)}\right\}, \quad \omega_{0}=\omega_{k_{0}} .
$$

Note that when $\tau=0$, (5) becomes

$$
\lambda^{3}+\left(a_{2}+b_{2}\right) \lambda^{2}+\left(a_{1}+b_{1}\right) \lambda+a_{0}+b_{0}=0 .
$$

Therefore, applying Lemmas 1 and 2 to (5), we obtain the following lemma.

Lemma 3. For the third-degree transcendental equation (5), one has the following results.

(i) If $r \geq 0$ and $\Delta=p^{2}-3 q \leqslant 0$, then all roots with positive real parts of (5) have the same sum to those of the polynomial equation (16) for all $\tau \geq 0$.

(ii) If either $r<0$ or $r \geq 0$ and $\Delta=p^{2}-3 q>0, z_{1}^{*}>0$, and $h\left(z_{1}^{*}\right)<0$, then all roots with positive real parts of (5) have the same sum to those of the polynomial equation (16) for $\tau \in\left[0, \tau_{0}\right)$.

Let $\lambda(\tau)=\alpha(\tau)+i \omega(\tau)$ be the root of (9) near $\tau=\tau_{k}^{(j)}$ satisfying

$$
\alpha\left(\tau_{k}^{(j)}\right)=0, \quad \omega\left(\tau_{k}^{(j)}\right)=\omega_{k} .
$$

Then it is easy to verify the following transversality condition.
Lemma 4. Suppose that $z_{k}=\omega_{k}^{2}$ and $h^{\prime}\left(z_{k}\right) \neq 0$, where $h(z)$ is defined by (15). Then $d\left(\operatorname{Re} \lambda\left(\tau_{k}^{(j)}\right)\right) / d \tau \neq 0$, and $d\left(\operatorname{Re} \lambda\left(\tau_{k}^{(j)}\right)\right) /$ $d \tau$ and $h^{\prime}\left(z_{k}\right)$ have the same sign.

Now, we study the characteristic equation (5) of system (4). Applying Lemmas 3 and 4 to (5), we have the following theorem.

Theorem 5. Let $\tau_{k}^{(j)}$ and $\omega_{0}, \tau_{0}$ be defined by (14) and (15), respectively. Then consider the following.

(i) If $r \geq 0$ and $\Delta=p^{2}-3 q \leq 0$, then all roots with positive real parts of (5) have the same sum to those of the polynomial equation (16) for all $\tau \geq 0$.

(ii) If either $r<0$ or $r \geq 0$ and $\Delta=p^{2}-3 q>0, z_{1}^{*}>0$, and $h\left(z_{1}^{*}\right)<0$, then $h(z)$ has at least one positive root $z_{k}$, and all roots with positive real parts of (5) have the same sum to those of the polynomial equation (16) for $\tau \in\left[0, \tau_{0}\right)$.

(iii) If the conditions of (ii) are satisfied and $h^{\prime}\left(z_{k}\right) \neq 0$, then system (2) exhibits Hopf bifurcation at the equilibrium $E_{0}$ for $\tau=\tau_{k}^{(j)}$.

\section{Stability and Direction of Bifurcating Periodic Orbits}

In the previous section, we obtain the conditions under which family periodic solutions bifurcate from the equilibrium $E_{0}$ at the critical value of $\tau$. As pointed by in Hassard et al. [17], it is interesting to determine the direction, stability, and period of these periodic solutions. Following the ideal of Hassard et al., we derive the explicit formulae for determining the properties of the Hopf bifurcation at the critical value of $\tau$ using the normal form and the center manifold theory. Throughout this section, we always assume that system (2) undergoes Hopf bifurcations at the equilibrium $E_{0}$ for $\tau=\tau_{k}$, and then $\pm i \omega_{k}$ is corresponding to purely imaginary roots of the characteristic equation at the equilibrium $E_{0}$.

Letting $\tau=\tau t, \tau=\tau_{k}+\mu$, system (2) is transformed into an FDE in $C=C\left([-1,0], R^{3}\right)$ as

$$
\dot{u}=L_{\mu}\left(u_{t}\right)+f\left(\mu, u_{t}\right),
$$

where $u(t)=(x(t), y(t), z(t))^{T} \in R^{3}$ and $L_{\mu}: C \rightarrow R, f:$ $R \times C \rightarrow R$ are given, respectively, by

$$
\begin{gathered}
L_{\mu}(\phi)=\left(\tau_{k}+\mu\right)\left(\begin{array}{ccc}
a & -1 & 0 \\
1 & K-b & -1 \\
a & 0 & -8
\end{array}\right)\left(\begin{array}{l}
\phi_{1}(0) \\
\phi_{2}(0) \\
\phi_{3}(0)
\end{array}\right) \\
+\left(\tau_{k}+\mu\right)\left(\begin{array}{ccc}
0 & 0 & 0 \\
0 & -K & 0 \\
0 & 0 & 0
\end{array}\right)\left(\begin{array}{l}
\phi_{1}(-1) \\
\phi_{2}(-1) \\
\phi_{3}(-1)
\end{array}\right), \\
f(\tau, \phi)=\left(\tau_{k}+\mu\right)\left(\begin{array}{c}
0 \\
0 \\
4 \phi_{2}(0) \phi_{3}(0)
\end{array}\right) .
\end{gathered}
$$


By the Riesz representation theorem, there exists a function $\eta(\theta, \mu)$ of bounded variation for $\theta \in[-1,0]$, such that

$$
L_{\mu} \phi=\int_{-1}^{0} d \eta(\theta, 0) \phi(\theta), \quad \text { for } \phi \in C .
$$

In fact, we can choose

$$
\begin{aligned}
\eta(\theta, \mu)= & \left(\tau_{k}+\mu\right)\left(\begin{array}{ccc}
a & -1 & 0 \\
1 & K-b & -1 \\
a & 0 & -8
\end{array}\right) \delta(\theta) \\
& -\left(\tau_{k}+\mu\right)\left(\begin{array}{ccc}
0 & 0 & 0 \\
0 & -K & 0 \\
0 & 0 & 0
\end{array}\right) \delta(\theta+1),
\end{aligned}
$$

where $\delta$ is Dirac-delta function.

For $\phi \in C^{1}\left([-1,0], R^{3}\right)$, define

$$
\begin{aligned}
& A(\mu) \phi= \begin{cases}\frac{d \phi(\theta)}{d \theta}, & \theta \in[-1,0), \\
\int_{-1}^{0} d \eta(\mu, s) \phi(s), & \theta=0 .\end{cases} \\
& R(\mu) \phi= \begin{cases}0, & \theta \in[-1,0), \\
f(\mu, \phi), & \theta=0 .\end{cases}
\end{aligned}
$$

Then, when $\theta=0$, system (18) is equivalent to

$$
\dot{u}_{t}=A(\mu) u_{t}+R(\mu) u_{t}
$$

where $u_{t}(\theta)=u(t+\theta)$ for $\theta \in[-1,0]$. For $\psi \in C^{1}([0,1]$, $\left.\left(R^{3}\right)^{*}\right)$, define

$$
A^{*} \psi(s)= \begin{cases}-\frac{d \psi(s)}{d s}, & s \in(0,1], \\ \int_{-1}^{0} d \eta^{T}(t, 0) \psi(-t), & s=0\end{cases}
$$

and a bilinear inner product

$$
\begin{aligned}
\langle\psi(s), \phi(\theta)\rangle= & \bar{\psi}(0) \phi(0) \\
& -\int_{-1}^{0} \int_{\xi=0}^{\theta} \bar{\psi}(\xi-\theta) d \eta(\theta) \phi(\xi) d \xi,
\end{aligned}
$$

where $\eta(\theta)=\eta(\theta, 0)$. Then $A=A(0)$ and $A^{*}$ are adjoint operators. By the discussion in Section 2, we know that $\pm i \omega_{k} \tau_{k}$ are eigenvalues of $A$. Thus, they are also eigenvalues of $A^{*}$. We first need to compute the eigenvector of $A$ and $A^{*}$ corresponding to $i \omega_{k} \tau_{k}$ and $-i \omega_{k} \tau_{k}$, respectively. Suppose that $q(\theta)=(1, \alpha, \beta)^{T} e^{i \theta \omega_{k} \tau_{k}}$ is the eigenvector of $A$ corresponding to $i \omega_{k} \tau_{k}$. Then $A q(\theta)=i \omega_{k} \tau_{k} q(\theta)$. It follows from the definition of $A$ and (19), (21), and (22) that

$$
\begin{gathered}
\tau_{k}\left(\begin{array}{ccc}
i \omega_{k}-a & 1 & 0 \\
-1 & i \omega_{k}-K+b+K e^{-i \omega_{k} \tau_{k}} & 1 \\
-a & 0 & i \omega_{k}+8
\end{array}\right) q(0) \\
=\left(\begin{array}{l}
0 \\
0 \\
0
\end{array}\right) .
\end{gathered}
$$

Thus, we can easily get

$$
q(0)=(1, \alpha, \beta)^{T}=\left(1, a-i \omega_{k}, \frac{a}{i \omega_{k}+8}\right)^{T} .
$$

Similarly, let $q^{*}(s)=D\left(1, \alpha^{*}, \beta^{*}\right) e^{i s \omega_{k} \tau_{k}}$ be the eigenvector of $A^{*}$ corresponding to $-i \omega_{k} \tau_{k}$. By the definition of $A^{*}$ and (19), (21), and (22), we can compute

$$
\begin{aligned}
q^{*}(s) & =D\left(1, \alpha^{*}, \beta^{*}\right) e^{i s \omega_{k} \tau_{k}} \\
& =D\left(1, \frac{-\left(i \omega_{k}+a\right)\left(i \omega_{k}-8\right)}{i \omega_{k}-8+a}, \frac{-i \omega_{k}-a}{i \omega_{k}-8+a}\right) e^{i s \omega_{k} \tau_{k}}
\end{aligned}
$$

In order to assure $\left\langle q^{*}(s), q(\theta)\right\rangle=1$, we need to determine the value of $D$. From (26), we have

$$
\begin{aligned}
\left\langle q^{*}(s), q(\theta)\right\rangle= & \bar{D}\left(1, \bar{\alpha}^{*}, \bar{\beta}^{*}\right)(1, \alpha, \beta)^{T} \\
& -\int_{-1}^{0} \int_{\xi=0}^{\theta} \bar{D}\left(1, \bar{\alpha}^{*}, \bar{\beta}^{*}\right) e^{-i(\xi-\theta) \omega_{k} \tau_{k}} d \eta \\
& \times(\theta)(1, \alpha, \beta)^{T} e^{i \xi \omega_{k} \tau_{k}} d \xi \\
= & \bar{D}\left\{1+\alpha \bar{\alpha}^{*}+\beta \bar{\beta}^{*}\right. \\
& -\int_{-1}^{0}\left(1, \bar{\alpha}^{*}, \bar{\beta}^{*}\right) \theta e^{i \theta \omega_{k} \tau_{k}} d \eta \\
& \left.\times(\theta)(1, \alpha, \beta)^{T}\right\} \\
= & \bar{D}\left\{1+\alpha \bar{\alpha}^{*}+\beta \bar{\beta}^{*}-K \tau_{k} \alpha \bar{\alpha}^{*} e^{-i \omega_{k} \tau_{k}}\right\} .
\end{aligned}
$$

Thus, we can choose $D$ as

$$
D=\frac{1}{1+\alpha \bar{\alpha}^{*}+\beta \bar{\beta}^{*}-K \tau_{k} \alpha \bar{\alpha}^{*} e^{i \omega_{k} \tau_{k}} .}
$$

In the following, we first compute the coordinates to describe the center manifold $C_{0}$ at $\mu=0$. Let $u_{t}$ be the solution of (18) when $\mu=0$. Define

$$
z(t)=\left\langle q^{*}, u_{t}\right\rangle, \quad W(t, \theta)=u_{t}(\theta)-2 \operatorname{Re}\{z(t) q(\theta)\} .
$$

On the center manifold $C_{0}$, we have

$$
\begin{aligned}
W(t, \theta)= & W(z(t), \bar{z}(t), \theta) \\
= & W_{20}(\theta) \frac{z^{2}}{2}+W_{11}(\theta) z \bar{z} \\
& +W_{02}(\theta) \frac{\bar{z}^{2}}{2}+W_{30}(\theta) \frac{z^{3}}{6}+\cdots,
\end{aligned}
$$

where $z$ and $\bar{z}$ are local coordinates for center manifold $C_{0}$ in the direction of $q$ and $q^{*}$. Note that $W$ is real if $u_{t}$ is real. 
We consider only real solutions. For the solution $u_{t} \in C_{0}$ of (18), since $\mu=0$, we have

$$
\begin{aligned}
\dot{z}= & i \omega_{k} \tau_{k} z \\
& +\left\langle q^{*}(\theta), f(0, W(z(t), \bar{z}(t), \theta)+2 \operatorname{Re}\{z(t) q(\theta)\})\right\rangle \\
= & i \omega_{k} \tau_{k} z \\
& \quad+\bar{q}^{*}(0) f(0, W(z(t), \bar{z}(t), 0)+2 \operatorname{Re}\{z(t) q(0)\}) \\
\triangleq & i \omega_{k} \tau_{k} z+\bar{q}^{*}(0) f_{0}(z, \bar{z})=i \omega_{k} \tau_{k} z+g(z, \bar{z}),
\end{aligned}
$$

where

$$
\begin{aligned}
g(z, \bar{z}) & =\bar{q}^{*}(0) f_{0}(z, \bar{z}) \\
& =g_{20}(\theta) \frac{z^{2}}{2}+g_{11}(\theta) z \bar{z}+g_{02}(\theta) \frac{\bar{z}^{2}}{2}+\cdots
\end{aligned}
$$

By (32), we have $u_{t}(\theta)=\left(u_{1 t}(\theta), u_{2 t}(\theta), u_{3 t}(\theta)\right)^{T}=W(t, \theta)+$ $z q(\theta)+\bar{z} \bar{q}(\theta)$, and then

$$
\begin{aligned}
u_{1 t}(0)= & z+\bar{z}+W_{20}^{(1)}(0) \frac{z^{2}}{2}+W_{11}^{(1)}(0) z \bar{z} \\
& +W_{02}^{(1)}(0) \frac{\bar{z}^{2}}{2}+o\left(|(z, \bar{z})|^{3}\right), \\
u_{2 t}(0)= & z \alpha+\bar{z} \bar{\alpha}+W_{20}^{(2)}(0) \frac{z^{2}}{2}+W_{11}^{(2)}(0) z \bar{z} \\
& +W_{02}^{(2)}(0) \frac{\bar{z}^{2}}{2}+o\left(|(z, \bar{z})|^{3}\right), \\
u_{3 t}(0)= & z \beta+\bar{z} \bar{\beta}+W_{20}^{(3)}(0) \frac{z^{2}}{2}+W_{11}^{(3)}(0) z \bar{z} \\
& +W_{02}^{(3)}(0) \frac{\bar{z}^{2}}{2}+o\left(|(z, \bar{z})|^{3}\right) .
\end{aligned}
$$

It follows together with (20) that

$$
\begin{aligned}
g(z, \bar{z})= & \bar{q}^{*}(0) f_{0}(z, \bar{z}) \\
= & \bar{D} \tau_{k}\left(1, \bar{\alpha}^{*}, \bar{\beta}^{*}\right)\left(\begin{array}{c}
0 \\
0 \\
4 u_{2 t}(0) u_{3 t}(0)
\end{array}\right) \\
= & 4 \bar{D} \tau_{k} \bar{\beta}^{*}\left[z \alpha+\bar{z} \bar{\alpha}+W_{20}^{(2)}(0) \frac{z^{2}}{2}+W_{11}^{(2)}(0) z \bar{z}\right. \\
& \times\left[\begin{array}{l}
\left.z \beta+\bar{z} \bar{\beta}+W_{20}^{(2)}(0) \frac{\bar{z}^{2}}{2}+o\left(|(z, \bar{z})|^{3}\right)\right] \\
2
\end{array}\right. \\
& \left.+W_{02}^{(3)}(0) \frac{z^{2}}{2}+o\left(|(z, \bar{z})|^{3}\right)\right] .
\end{aligned}
$$

Comparing the coefficients with (35), we have

$$
\begin{gathered}
g_{20}=8 \bar{D} \tau_{k} \bar{\beta}^{*} \alpha \beta, \\
g_{11}=8 \bar{D} \tau_{k} \bar{\beta}^{*}(\alpha \bar{\beta}+\bar{\alpha} \beta), \\
g_{02}=8 \bar{D} \tau_{k} \bar{\beta}^{*} \bar{\alpha} \bar{\beta} \\
g_{21}=4 \bar{D} \tau_{k} \bar{\beta}^{*}\left[2 \alpha W_{11}^{(3)}(0)+\bar{\alpha} W_{20}^{(3)}(0)\right. \\
\left.+\bar{\beta} W_{20}^{(2)}(0)+2 \beta W_{11}^{(2)}(0)\right] .
\end{gathered}
$$

In order to determine $g_{21}$, in the sequel, we need to compute $W_{20}(\theta)$ and $W_{11}(\theta)$. From (24) and (32), we have

$$
\begin{aligned}
\dot{W} & =\dot{u}_{t}-\dot{z} q-\dot{\bar{z}} \bar{q} \\
& = \begin{cases}A W-2 \operatorname{Re}\left\{\bar{q}^{*}(0) f_{0} q(\theta)\right\}, & \theta \in[0,1) \\
A W-2 \operatorname{Re}\left\{\bar{q}^{*}(0) f_{0} q(\theta)\right\}+f_{0}, & \theta=0\end{cases} \\
& \triangleq A W+H(z, \bar{z}, \theta),
\end{aligned}
$$

where

$$
H(z, \bar{z}, \theta)=H_{20}(\theta) \frac{z^{2}}{2}+H_{11}(\theta) z \bar{z}+H_{02}(\theta) \frac{\bar{z}^{2}}{2}+\cdots
$$

Notice that near the origin on the center manifold $C_{0}$, we have

$$
\dot{W}=W_{z} \dot{z}+W_{\bar{z}} \dot{\bar{z}}
$$

Thus, we have

$$
\begin{gathered}
\left(A-2 i \tau_{k} \omega_{k} I\right) W_{20}(\theta)=-H_{20}(\theta), \\
A W_{11}(\theta)=-H_{11}(\theta) .
\end{gathered}
$$

Since (39), for $\theta \in[-1,0)$, we have

$$
\begin{aligned}
H(z, \bar{z}, \theta) & =-\bar{q}^{*}(0) f_{0} q(\theta)-q^{*}(0) \bar{f}_{0} \bar{q}(\theta) \\
& =-g q(\theta)-\bar{g} \bar{q}(\theta) .
\end{aligned}
$$

Comparing the coefficients with (40) gives that

$$
\begin{aligned}
& H_{20}(\theta)=-g_{20} q(\theta)-\bar{g}_{02} \bar{q}(\theta), \\
& H_{11}(\theta)=-g_{11} q(\theta)-\bar{g}_{11} \bar{q}(\theta) .
\end{aligned}
$$

From (43), (46), and the definition of $A$, we can get

$$
\dot{W}_{20}(\theta)=2 i \tau_{k} \omega_{k} W_{20}(\theta)+g_{20} q(\theta)+\bar{g}_{02} \bar{q}(\theta) \text {. }
$$

Noticing that $q(\theta)=q(0) e^{i \tau_{k} \omega_{k} \theta}$, we have

$$
\begin{aligned}
W_{20}(\theta)= & \frac{i g_{20}}{\tau_{k} \omega_{k}} q(0) e^{i \tau_{k} \omega_{k} \theta}+\frac{i \bar{g}_{02}}{3 \tau_{k} \omega_{k}} \bar{q}(0) e^{-i \tau_{k} \omega_{k} \theta} \\
& +E_{1} e^{2 i \tau_{k} \omega_{k} \theta}
\end{aligned}
$$




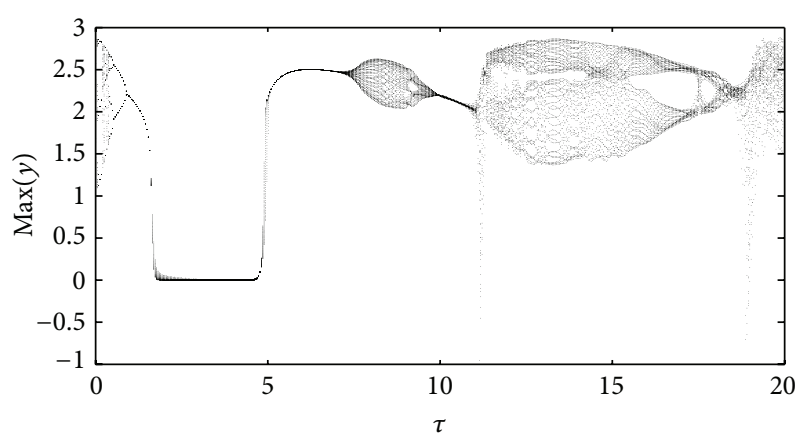

FIgURE 2: The bifurcation graph with $\tau$ when $K=-1$.

where $E_{1}=\left(E_{1}^{(1)}, E_{1}^{(2)}, E_{1}^{(3)}\right) \in R^{3}$ is a constant vector. In the same way, we can also obtain

$$
W_{11}(\theta)=-\frac{i g_{11}}{\tau_{k} \omega_{k}} q(0) e^{i \tau_{k} \omega_{k} \theta}+\frac{i \bar{g}_{11}}{\tau_{k} \omega_{k}} \bar{q}(0) e^{-i \tau_{k} \omega_{k} \theta}+E_{2},
$$

where $E_{2}=\left(E_{2}^{(1)}, E_{2}^{(2)}, E_{2}^{(3)}\right) \in R^{3}$ is also a constant vector.

In what follows, we will seek appropriate $E_{1}$ and $E_{2}$. From the definition of $A$ and (42), we obtain

$$
\begin{gathered}
\int_{-1}^{0} d \eta(\theta) W_{20}(\theta)=2 i \tau_{k} \omega_{k} W_{20}(0)-H_{20}(0), \\
\int_{-1}^{0} d \eta(\theta) W_{11}(\theta)=-H_{11}(0),
\end{gathered}
$$

where $\eta(\theta)=\eta(0, \theta)$. From (39) and (40), we have

$$
\begin{gathered}
H_{20}(0)=-g_{20} q(0)-\bar{g}_{02} \bar{q}(0)+2 \tau_{k}\left(\begin{array}{c}
0 \\
0 \\
4 \alpha \beta
\end{array}\right), \\
H_{11}(0)=-g_{11} q(0)-\bar{g}_{11} \bar{q}(0)+2 \tau_{k}\left(\begin{array}{c}
0 \\
0 \\
4 \operatorname{Re}\{\alpha \bar{\beta}\}
\end{array}\right) .
\end{gathered}
$$

Substituting (46) and (50) into (48) and noticing that

$$
\begin{gathered}
\left(i \omega_{k} \tau_{k} I-\int_{-1}^{0} e^{i \omega_{k} \tau_{k} \theta} d \eta(\theta)\right) q(0)=0, \\
\left(-i \omega_{k} \tau_{k} I-\int_{-1}^{0} e^{-i \omega_{k} \tau_{k} \theta} d \eta(\theta)\right) \bar{q}(0)=0,
\end{gathered}
$$

we obtain

$$
\left(2 i \omega_{k} \tau_{k} I-\int_{-1}^{0} e^{2 i \omega_{k} \tau_{k} \theta} d \eta(\theta)\right) E_{1}=2 \tau_{k}\left(\begin{array}{c}
0 \\
0 \\
4 \alpha \beta
\end{array}\right),
$$

which leads to

$$
\begin{aligned}
& \left(\begin{array}{ccc}
2 i \omega_{k}-a & 1 & 0 \\
-1 & 2 i \omega_{k}-K+b+K e^{-2 i \omega_{k} \tau_{k}} & 1 \\
-a & 0 & 2 i \omega_{k}+8
\end{array}\right) E_{1} \\
& \quad=2\left(\begin{array}{c}
0 \\
0 \\
4 \alpha \beta
\end{array}\right) .
\end{aligned}
$$

It follows that

$$
\begin{gathered}
E_{1}^{(1)}=\frac{8}{A} \alpha \beta \\
E_{1}^{(2)}=\frac{8}{A} \alpha \beta\left(2 i \omega_{k}-a\right), \\
E_{1}^{(3)}=\frac{8}{A} \alpha \beta\left[\left(2 i \omega_{k}-a\right)\left(2 i \omega_{k}-K+b+K e^{-2 i \omega_{k} \tau_{k}}\right)+1\right],
\end{gathered}
$$

where

$$
\begin{aligned}
A= & \left(2 i \omega_{k}+8\right) \\
& \times\left[\left(2 i \omega_{k}-a\right)\left(2 i \omega_{k}-K+b+K e^{-2 i \omega_{k} \tau_{k}}\right)+1\right]-a .
\end{aligned}
$$

Similarly, substituting (47) and (51) into (49), we can get

$$
\left(\begin{array}{lll}
-a & 1 & 0 \\
-1 & b & 1 \\
-a & 0 & 8
\end{array}\right) E_{2}=2\left(\begin{array}{c}
0 \\
0 \\
4 \operatorname{Re}\{\alpha \bar{\beta}\}
\end{array}\right)
$$

Thus, we have

$$
\begin{gathered}
E_{2}^{(1)}=\frac{8}{B} \operatorname{Re}\{\alpha \bar{\beta}\}, \\
E_{2}^{(2)}=\frac{8}{B} \operatorname{Re}\{\alpha \bar{\beta}\}, \\
E_{2}^{(3)}=\frac{8}{B} \operatorname{Re}\{\alpha \bar{\beta}\}(1-a b),
\end{gathered}
$$

where

$$
B=8-a-8 a b .
$$

Thus, we can determine $W_{20}(\theta)$ and $W_{11}(\theta)$. Furthermore, we can determine each $g_{i j}$. Therefore, each $g_{i j}$ is determined by the parameters and delay in (2). Thus, we can compute the following values:

$$
\begin{gathered}
c_{1}(0)=\frac{i}{2 \omega_{k} \tau_{k}}\left(g_{20} g_{11}-2\left|g_{11}\right|^{2}-\frac{1}{3}\left|g_{02}\right|^{2}\right)+\frac{g_{21}}{2}, \\
\mu_{2}=-\frac{\operatorname{Re}\left\{c_{1}(0)\right\}}{\operatorname{Re}\left\{\lambda^{\prime}\left(\tau_{k}\right)\right\}}, \\
T_{2}=-\frac{\operatorname{Im}\left\{c_{1}(0)\right\}+\mu_{2} \operatorname{Im}\left\{\lambda^{\prime}\left(\tau_{k}\right)\right\}}{\omega_{k} \tau_{k}}, \\
\beta_{2}=2 \operatorname{Re}\left\{c_{1}(0)\right\},
\end{gathered}
$$

which determine the quantities of bifurcating periodic solutions in the center manifold at the critical value $\tau_{k}$; that is, $\mu_{2}$ determines the directions of the Hopf bifurcation: if $\mu_{2}>0$ (< 0 ), then the Hopf bifurcation is supercritical (subcritical), and the bifurcation exists for $\tau>\tau_{0}\left(<\tau_{0}\right) ; \beta_{2}$ determines the stability of the bifurcation periodic solutions: the bifurcating periodic solutions are stable (unstable) if $\beta_{2}<0(>0)$; and $T_{2}$ determines the period of the bifurcating periodic solutions: the period increases (decreases) if $T_{2}>0(<0)$. 

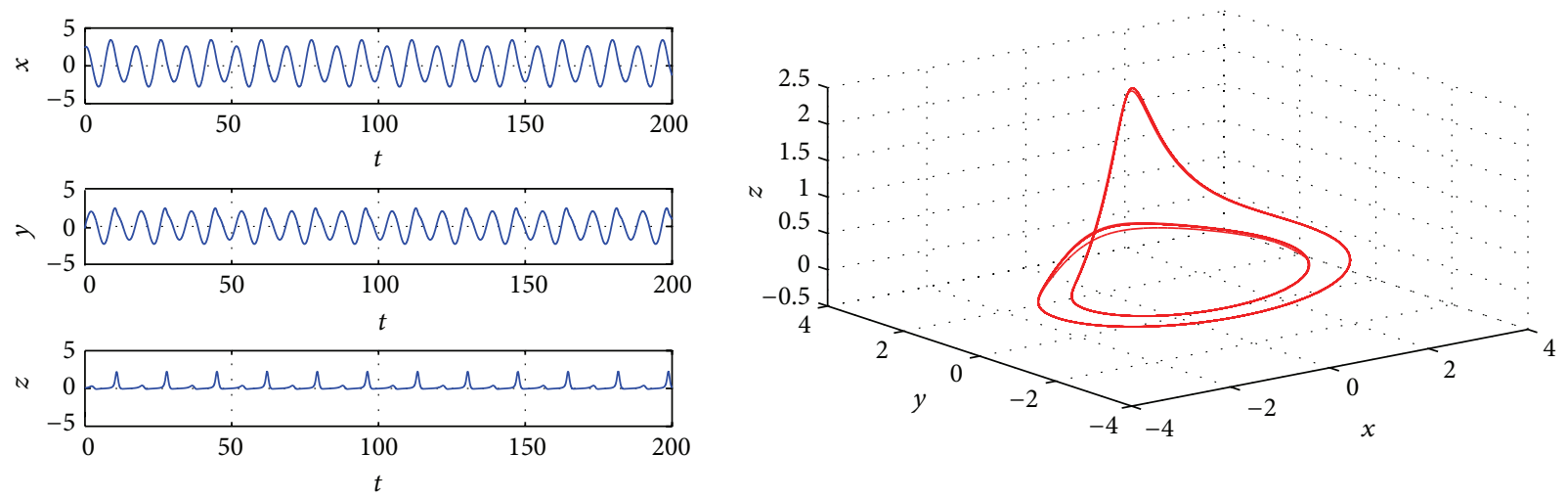

FIgURE 3: The trajectories and phase graphs of system (61) with $\tau=0.7362$ when $K=-1$.
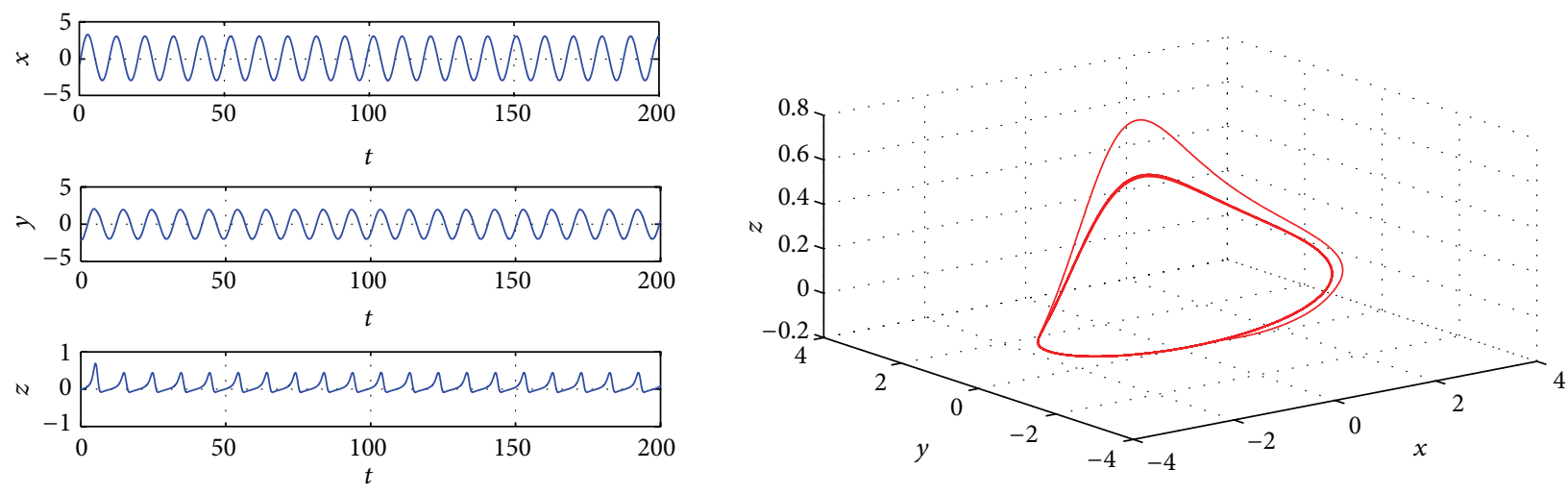

FIGURE 4: The trajectories and phase graphs of system (61) with $\tau=1.3497$ when $K=-1$.

\section{Numerical Simulations and Application to Control of Chaos}

In this section, we apply the results we get in the previous sections to system (61) for the purpose of control of chaos. From Section 2, we know that under certain conditions, a family of periodic solutions bifurcates from the steady states of system (61) at some critical values of $\tau$, and the stability of the steady state may be changed along with the increase of $\tau$. If the bifurcating periodic solution is orbitally asymptotically stable or some steady state becomes local stable, then chaos may vanish. Following this idea, we consider the following delayed feedback control system for an autonomy system:

$$
\begin{gathered}
\dot{x}=0.2 x-y, \\
\dot{y}=x-0.02 y-z+K(y(t)-y(t-\tau)), \\
\dot{z}=0.2 x+4 z(y-2),
\end{gathered}
$$

which has two equilibriums, $E_{0}(0,0,0)$ and $E^{*}(9.749,1.9498$, 9.710). When $\tau=0$ or $K=0, E_{0}$ is unstable and system
(61) is chaotic (see Figure 1). The corresponding characteristic equation of system (61) at $E_{0}$ is

$$
\begin{gathered}
\lambda^{3}+(7.82-K) \lambda^{2}-(0.444+7.8 K) \lambda+7.768 \\
+1.6 K+\left(\lambda^{2}+7.8 \lambda-1.6\right) K e^{-\lambda \tau}=0
\end{gathered}
$$

When $\tau=0$, (62) has one negative root and a pair of complex roots with positive parts. By the discussion of Section 2, we can get $p=62.0404-0.04 K, q=-121.2944-$ $2.5616 K$, and $r=60.3418+24.8576 K$, and then we can compute $\Delta=4212.9+2.7216 K+0.0016 K^{2}$, and it is easy to know that $\triangle>0$ and when $K \geq-2.4275, z_{1}^{*}>0$ always holds. Then, by Theorem 5, we have the following conclusion.

Conclusion 1. If $K<-2.4275$ or $K \geq-2.4275$ and $h\left(z_{1}^{*}\right)<0$, then there exist some $\tau_{0}>0$, such that when $\tau \in\left[0, \tau_{0}\right), E_{0}$ is always unstable.

For example, we fix $K=-1$. Through computing, we can get $z_{1}^{*}=1.0693>0$ and $h\left(z_{1}^{*}\right)=-19.2713<0$. Furthermore, we can compute 

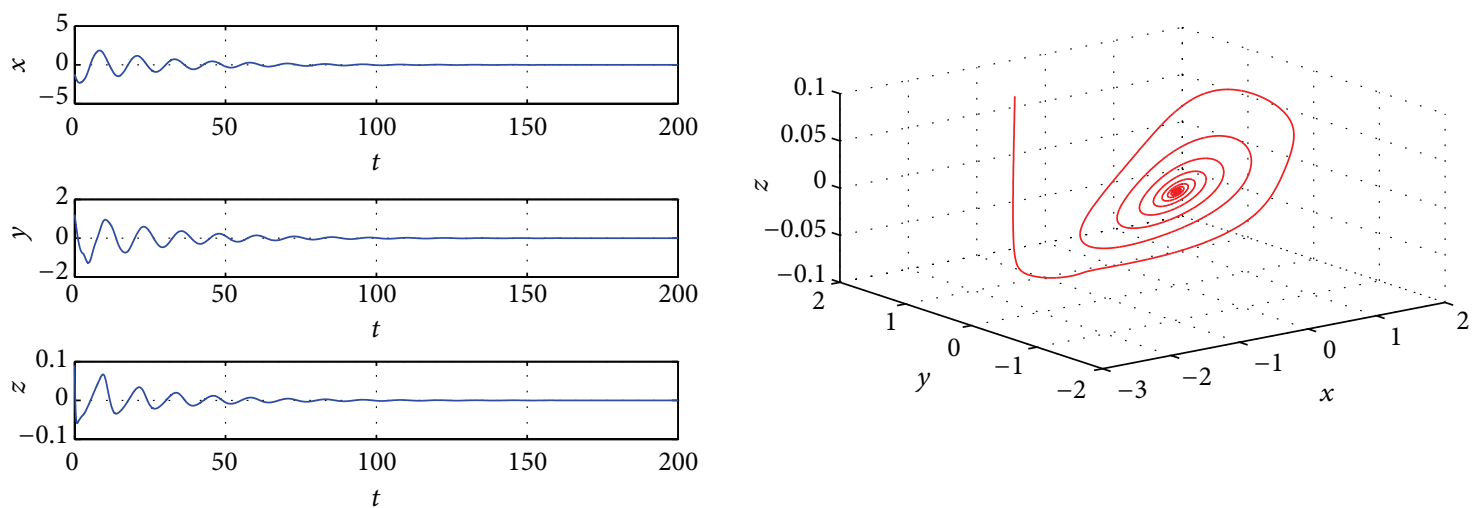

FIgURE 5: The trajectories and phase graphs of system (61) with $\tau=2.6815$ when $K=-1$.
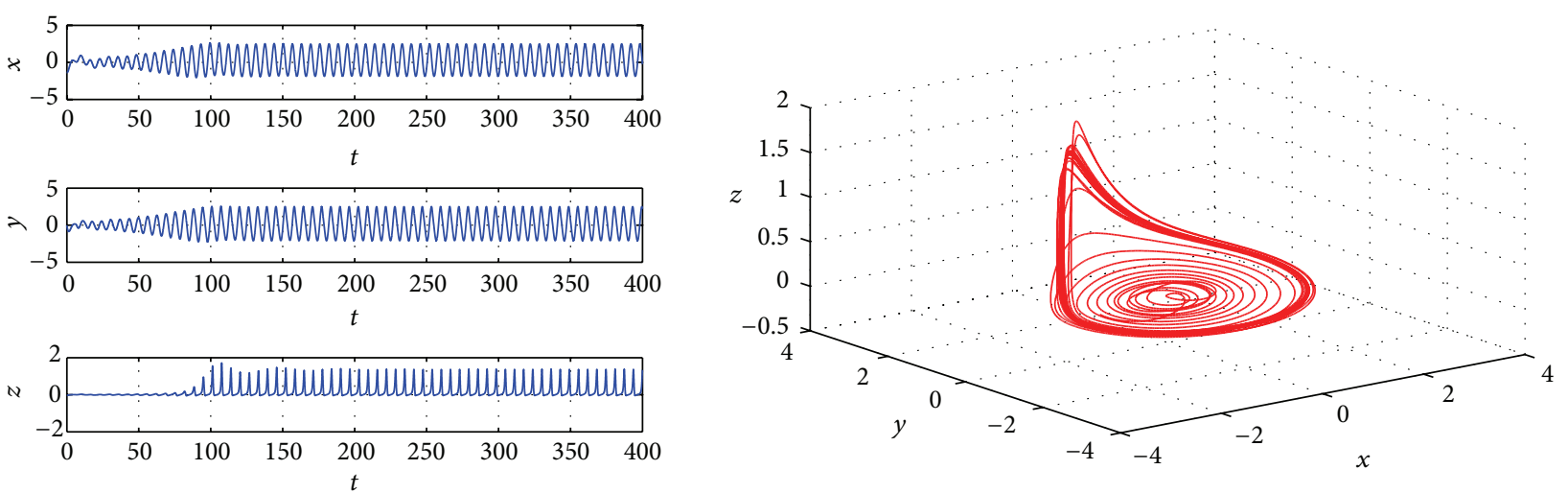

Figure 6: The trajectories and phase graphs of system (61) with $\tau=6.3804$ when $K=-1$.

$$
\begin{array}{rlrl}
z_{1} & \doteq 0.3714185849, & \omega_{1} & \doteq 0.6094, \\
\tau_{1}^{(j)}=1.6243+\frac{2 j \pi}{\omega_{1}}, & h^{\prime}\left(z_{1}\right)=-71.3282, \\
z_{2} \doteq 1.494030027, & \omega_{2} \doteq 1.2223, \\
\tau_{2}^{(j)}=0.3768+\frac{2 j \pi}{\omega_{2}}, & h^{\prime}\left(z_{2}\right)=72.5266 .
\end{array}
$$

Thus, from Lemma 4, we have

$$
\frac{\operatorname{Re} \lambda\left(\tau_{1}^{(j)}\right)}{d \tau}<0, \quad \frac{\operatorname{Re} \lambda\left(\tau_{2}^{(j)}\right)}{d \tau}>0 .
$$

In addition, notice that

$$
\begin{aligned}
\tau_{2}^{(0)} & =0.3768<\tau_{1}^{(0)}=1.6243<\tau_{2}^{(1)} \\
& =5.5173<\tau_{2}^{(2)}=10.6577<\tau_{1}^{(1)} \\
& =11.9340<\tau_{2}^{(3)}=15.7982<\cdots
\end{aligned}
$$

Thus, from (64) and (65), we have the following conclusion about the stability of the zero equilibrium of system (61) and Hopf bifurcations.
Conclusion 2. Suppose that $\tau_{k}^{(j)}, k=1,2, j=0,1, \ldots$, is defined by (63).

(i) When $\tau \in\left[0, \tau_{1}^{(0)}\right) \cup\left(\tau_{2}^{(1)},+\infty\right)$, the equilibrium $E_{0}$ is unstable (see Figures 3, 4, 6, 7, and 8).

(ii) When $\tau \in\left(\tau_{1}^{(0)}, \tau_{2}^{(1)}\right)$, the equilibrium $E_{0}$ is asymptotically stable (see Figure 5).

(iii) System (61) undergoes a Hopf bifurcation at the equilibrium $E_{0}$ when $\tau=\tau_{k}^{(j)}$ (see Figures 3, 4, and 6).

Thus, if we take $\tau \in\left(\tau_{1}^{(0)}, \tau_{2}^{(1)}\right)$, the stability of zero equilibrium of the system will change from unstable to stable. For this example, there are more complicated dynamical phenomena occurs. Bifurcation diagram is plotted with the parameter $\tau$ when $K=-1$ (Figure 2). In Figure 2, we can see that chaos vanishes via a period-doubling bifurcation when $\tau$ varying from 0 to 0.3768 . Figure 3 shows that, when $\tau=$ 0.7362 , a period- 2 orbits bifurcating from zero equilibrium. Figure 4 shows when $\tau=1.3497$, a period- 1 periodical orbits bifurcating from zero equilibrium. In Figure 2, a stable window is obtained from $\tau=1.6243$ to $\tau=5.5173$, which is demonstrated by Figure 5 with $\tau=2.6815$. When $\tau>5.5173$, Figure 2 shows that periodic orbits and strange attractors also 

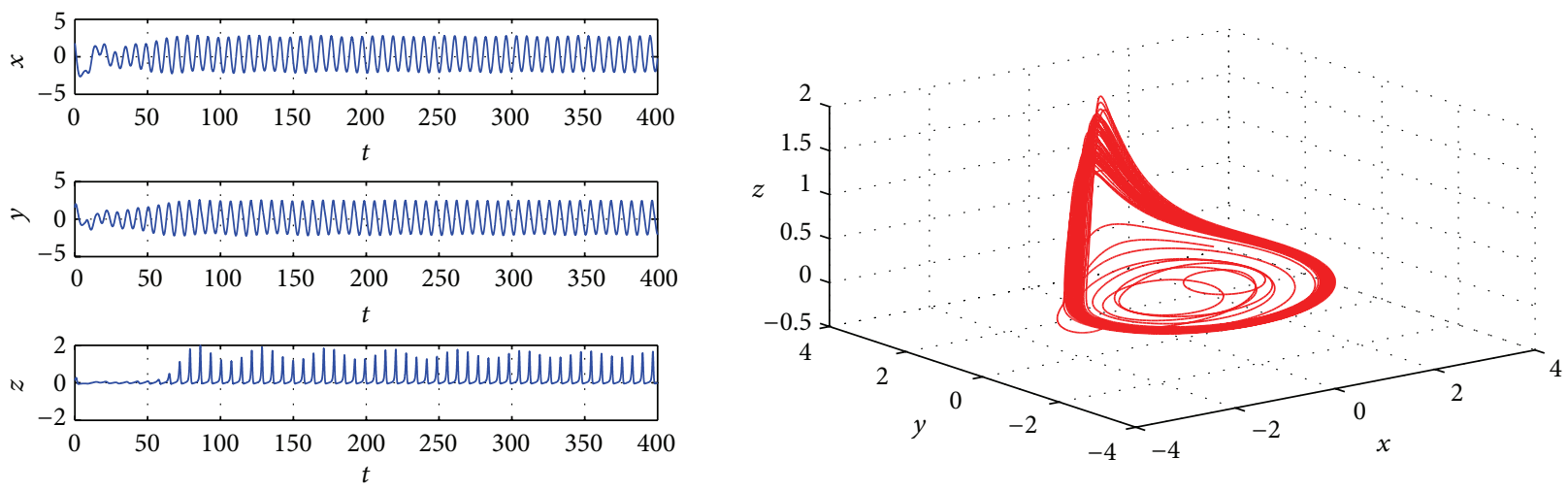

FIgURE 7: The trajectories and phase graphs of system (61) with $\tau=7.3006$ when $K=-1$.
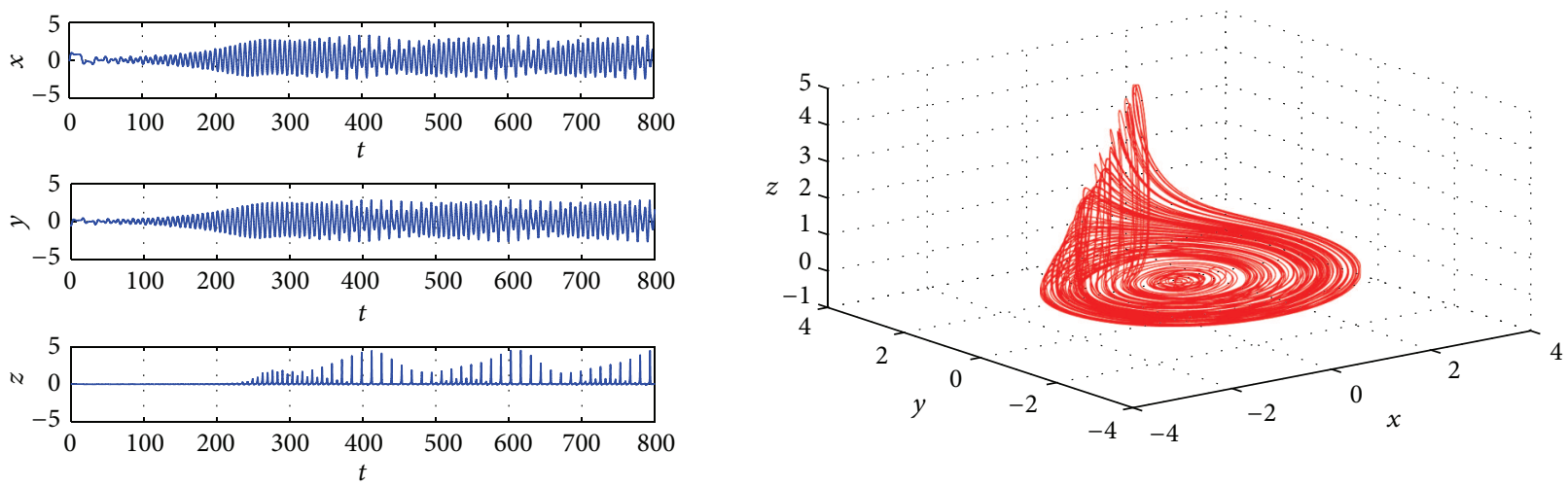

FIgURE 8: The trajectories and phase graphs of system (61) with $\tau=13.7423$ when $K=-1$.

occur, and system (61) through Hopf bifurcation route still goes to chaos, and it is demonstrated by Figures 6-8.

\section{Discussion}

In this paper, an autonomy system with time-delayed feedback is studied by using the theory of functional differential equation and Hassard's method. Illustrating with numerical simulations, we show that delayed feedback controller (DFC) is an effective method for chaos control.

In fact, system (2) has more complicated dynamical behaves. From Figure 2, we can see that chaos degeneration process, the orbits of system (2) continuously degenerate to a periodic solution region through reverse period-doubling bifurcation. With the increasing of $\tau$, chaos occurs again through Hopf bifurcation route. However, the numerical simulations indicate that there were points of similarity between this route to chaos and quasiperiodicity route to chaos [18]; in our future research, we will consider this topic. And in this paper, we only give detailed analysis on the trivial equilibrium $E_{0}$; the detailed analysis of the interior equilibrium $E^{*}$ is the second interested topic in our future research.

\section{Acknowledgments}

The authors would like to thank the anonymous referee for the very helpful suggestions and comments which led to the improvement of the original paper. And this work is supported by 2013 Scientific Research Project of Beifang University of Nationalities (2013XYZ021), Institute of Information and System Computation Science of Beifang University (13xyb01), Science and Technology Department of Henan Province (122300410417), and Education Department of Henan Province (13A110108).

\section{References}

[1] G. Corso and F. B. Rizzato, "Hamiltonian bifurcations leading to chaos in low-energy relativistic wave- particle system," Physica D, vol. 80, pp. 296-306, 1995.

[2] M. Saleem and T. Agrawal, "Chaos in a tumor growth model with delayed responses of the immune system," Journal of Applied Mathematics, vol. 2012, Article ID 891095, 16 pages, 2012.

[3] U. Kumar, "A retrospection of chaotic phenomene in electrical systems," Active and Passive Electronic Components, vol. 21, pp. $1-15,1998$. 
[4] Z. Chen, X. Zhang, and Q. Bi, "Bifurcations and chaos of coupled electrical circuits," Nonlinear Analysis, vol. 9, no. 3, pp. 1158-1168, 2008.

[5] S. Hallegatte, M. Ghil, P. Dumas, and J. Hourcade, "Business cycles, bifurcations and chaos in a neo-classical model with investment dynamics," Journal of Economic Behavior and Organization, vol. 67, pp. 57-77, 2008.

[6] D. Ghosh, A. R. Chowdhury, and P. Saha, "Bifurcation continuation, chaos and chaos control in nonlinear Bloch system," Communications in Nonlinear Science and Numerical Simulation, vol. 13, no. 8, pp. 1461-1471, 2008.

[7] A. El-Gohary and A. S. Al-Ruzaiza, "Chaos and adaptive control in two prey, one predator system with nonlinear feedback," Chaos, Solitons and Fractals, vol. 34, no. 2, pp. 443-453, 2007.

[8] H. Salarieh and A. Alasty, "Control of stochastic chaos using sliding mode method," Journal of Computational and Applied Mathematics, vol. 225, no. 1, pp. 135-145, 2009.

[9] J. Guan, "Bifurcation analysis and chaos control in genesio system with delayed feedback," ISRN Mathematical Physics, vol. 2012, Article ID 843962, 12 pages, 2012.

[10] H.-T. Yau and C.-S. Shieh, "Chaos synchronization using fuzzy logic controller," Nonlinear Analysis, vol. 9, no. 4, pp. 1800-1810, 2008.

[11] N. Vasegh and A. Sedigh, "Delayed feedback control of timedelayed chaotic systems: analytical approach at Hopf bifurcation," Physics Letters A, vol. 372, pp. 5110-5114, 2008.

[12] J. Lu, Z. Ma, and L. Li, "Double delayed feedback control for the stabilization of unstable steady states in chaotic systems," Communications in Nonlinear Science and Numerical Simulation, vol. 14, no. 7, pp. 3037-3045, 2009.

[13] M. Bleich and J. Socolar, "Stability of periodic orbits controlled by time-delay feedback," Physics Letters A, vol. 210, pp. 87-94, 1996.

[14] Y. Ding, W. Jiang, and H. Wang, "Delayed feedback control and bifurcation analysis of Rossler chaotic system," Nonlinear Dynamics, vol. 61, no. 4, pp. 707-715, 2010.

[15] Y. Song and J. Wei, "Bifurcation analysis for Chen's system with delayed feedback and its application to control of chaos," Chaos, Solitons and Fractals, vol. 22, no. 1, pp. 75-91, 2004.

[16] S. Ruan and J. Wei, "On the zeros of transcendental functions with applications to stability of delay differential equations with two delays," Dynamics of Continuous, Discrete and Impulsive Systems A, vol. 10, no. 6, pp. 863-874, 2003.

[17] B. D. Hassard, N. D. Kazarinoff, and Y. H. Wan, Theory and Applications of Hopf Bifurcation, vol. 41, Cambridge University Press, Cambridge, UK, 1981.

[18] I. Fuente, L. Martinez, J. Veguillas, and J. Aguirregabiria, "Quasiperiodicity route to chaos in a biochemical system," Biophysical Journal, vol. 71, pp. 2375-2379, 1998. 


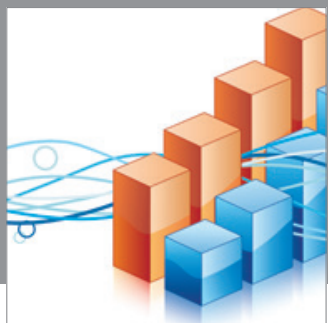

Advances in

Operations Research

mansans

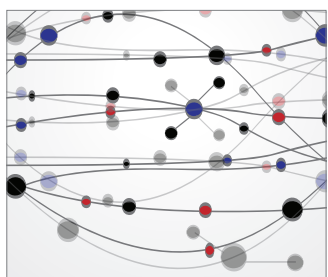

The Scientific World Journal
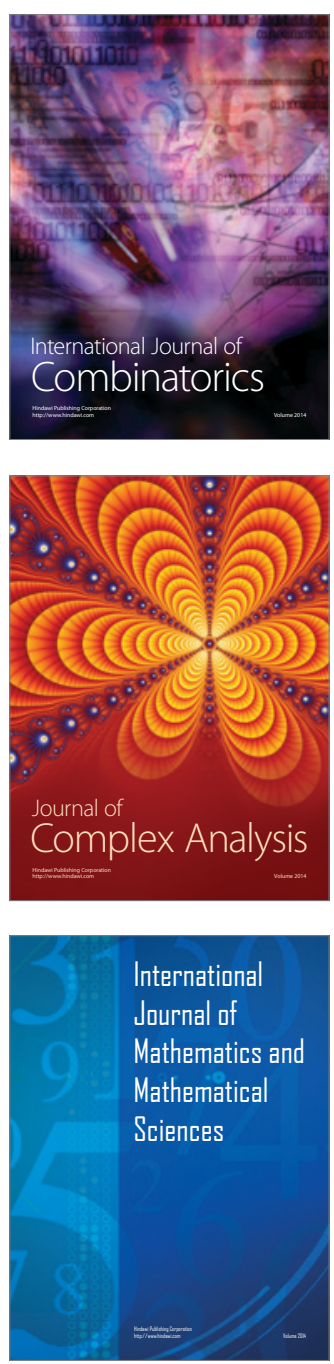
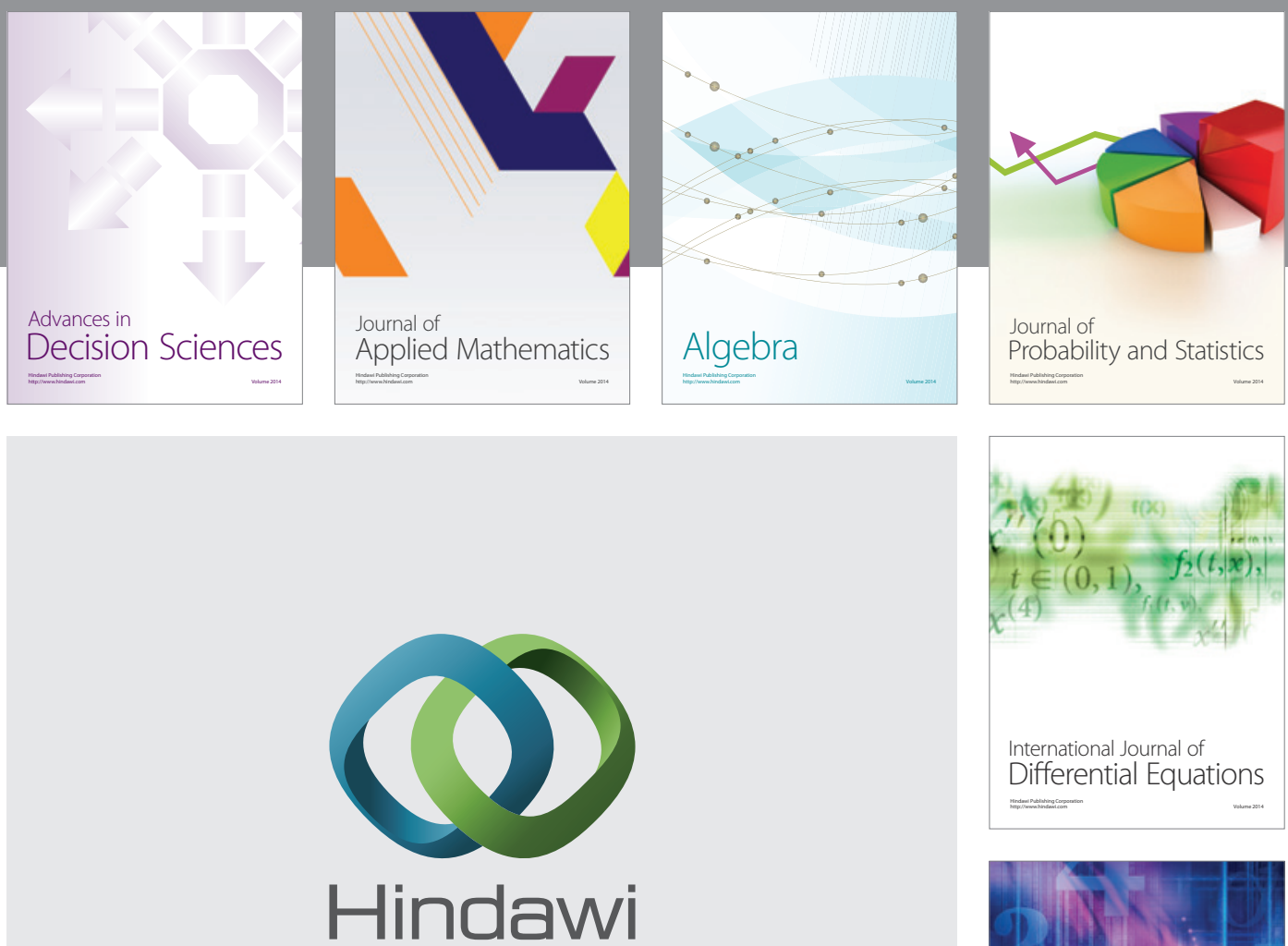

Submit your manuscripts at http://www.hindawi.com
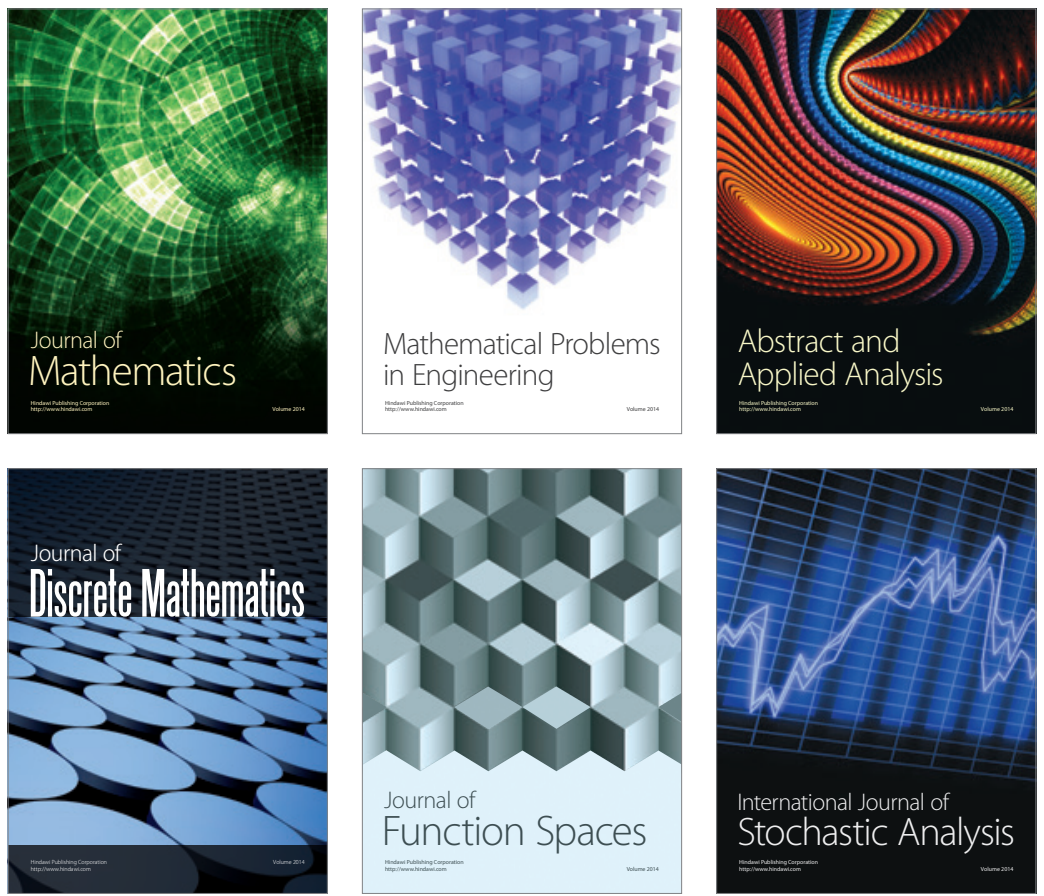

Journal of

Function Spaces

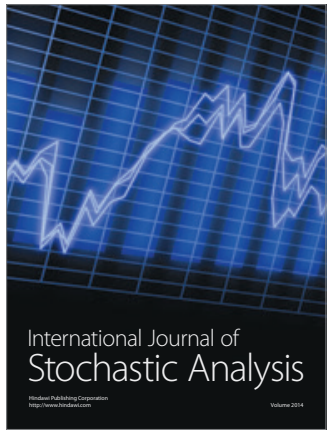

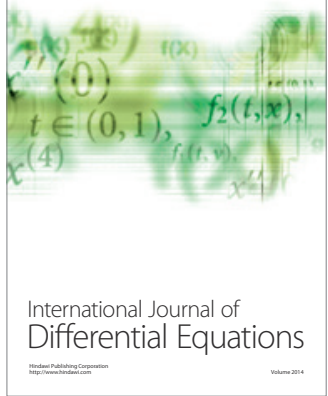
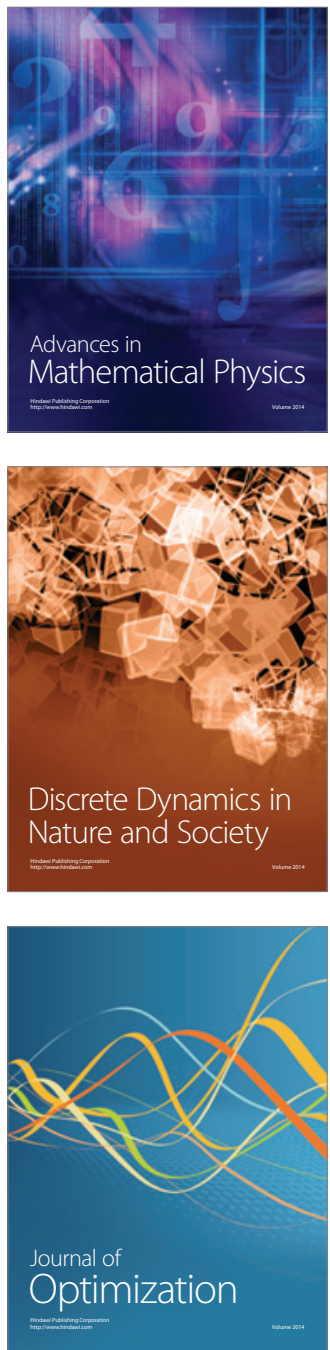